General Thoracic

Surgery

\title{
Comparison of mutational changes in involved N1 lymph nodes with those in primary tumors in stage II non-small cell lung cancer: A pilot study
}

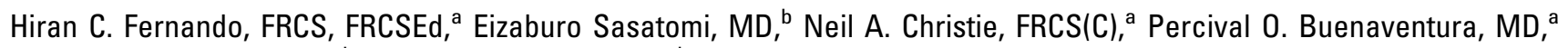
Sydney D. Finkelstein, MD, ${ }^{\mathrm{b}}$ Samuel A. Yousem, MD, ${ }^{\mathrm{b}}$ Ryan Soose, ${ }^{\mathrm{a}}$ John M. Close, MA, PMSD, ${ }^{\mathrm{c}}$ and James D. Luketich, $\mathrm{MD}^{\mathrm{a}}$

From the Division of Thoracic and Foregut Surgery, ${ }^{\mathrm{a}}$ the Department of Pathology, ${ }^{\mathrm{b}}$ and the Department of Dental Public Health, ${ }^{\mathrm{c}}$ University of Pittsburgh Medical Center Health System, UPMC Presbyterian, Pittsburgh, $\mathrm{Pa}$.

Read at the Eighty-second Annual Meeting of The American Association for Thoracic Surgery, Washington, DC, May 5-8, 2002.

Received for publication May 10, 2002; revisions requested Sept 10, 2002; revisions received Nov 30, 2002; accepted for publication Feb 3, 2003.

Address for reprints: Hiran C. Fernando, FRCS, Division Thoracic Surgery, UPMC Presbyterian, 200 Lothrop St, Suite C-800, Pittsburgh, PA 15213 (E-mail: fernandohc@msx.upmc.edu).

J Thorac Cardiovasc Surg 2004;127:87-91 $0022-5223 / \$ 30.00$

Copyright () 2004 by The American Association for Thoracic Surgery

doi:10.1016/j.jtcvs.2003.02.001
Objectives: Surgical resection is the standard treatment for stage II non-small cell lung cancer, but recurrence rates approach $60 \%$. This study compared mutational changes in involved lymph nodes and primary tumors from patients with stage II non-small cell lung cancer to determine whether risk factors for recurrence could be identified.

Methods: Forty patients with resected stage II non-small cell lung cancer (excluding T3 N0 disease) were studied. Microdissection was performed on primary tumors and lymph nodes. Analysis was performed across 9 genomic loci by using polymerase chain reaction amplification. The ratio of fractional allelic loss between involved lymph nodes and primary tumors was used to stratify patients into high-risk (fractional allelic loss ratio of $\geq 1$ ) and low-risk (fractional allelic loss ratio of $<1$ ) groups.

Results: The median age of the patients was 68 years (range, 42-85 years). Median follow-up was 30 months. Fractional allelic loss was greater in patients with squamous carcinomas compared with that in adenocarcinomas, but survival was similar (35 vs 39 months). The median survival was 35 months in high-risk patients and was not reached in low-risk patients $(P=.3)$. Disease-free survival was 24 months in high-risk patients and was not reached in low-risk patients $(P=.35)$. In the subset with adenocarcinoma $(\mathrm{n}=18)$, median survival was 24 months in the high-risk group; no deaths occurred in low-risk patients $(P=.01)$. Also, diseasefree survival was 14 months in high-risk patients and was not reached in the low-risk patients $(P=.05)$.

Conclusions: Squamous cancers demonstrate greater mutational changes than adenocarcinomas; this does not affect outcome. The patients with low-risk adenocarcinomas demonstrated superior outcomes compared with those of other patients. These results should be confirmed in larger studies.

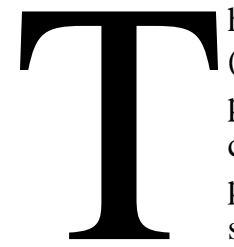

he current standard of care for stage II non-small cell lung cancer (NSCLC) is pulmonary resection. However, overall survival for this patient group is less than $40 \%$. $^{1}$ Adjuvant therapy, using radiation or chemotherapy trials, has generally failed to demonstrate any improvement in survival for this subset of NSCLC over the benefits of surgical intervention alone. ${ }^{2,3}$ Although providing some insight into the prognosis and guidelines for therapy, the staging system currently in place is 
TABLE 1. Relationships of microsatellite, chromosomal locus, and gene

\begin{tabular}{lcl}
\hline Microsatellite & $\begin{array}{c}\text { Chromosomal } \\
\text { locus }\end{array}$ & Gene \\
\hline D1S407, MYCL1 & $1 \mathrm{p} 34$ & L-myc \\
D3S1539, D3S2303 & $3 \mathrm{p} 26$ & OGG1 \\
D5S592, D5S615, & $5 \mathrm{q} 21$ & $A P C$ \\
$\quad$ MCC.E10 & & \\
D7S1530 & $7 \mathrm{q} 31$ & $c-M E T$ \\
D8S373 & $8 \mathrm{p} 24$ & $c-m y c$ \\
D9S254, D9S251 & $9 \mathrm{p} 21$ & $p 16$ \\
D10S1173, D10S520 & $10 \mathrm{q} 23$ & $P T E N$ \\
D17S1163, TP53 & $17 \mathrm{p} 13$ & $p 53$ \\
D18S814 & $18 \mathrm{q} 21$ & $D C C$ \\
\hline
\end{tabular}

inadequate. Molecular studies of NSCLC have been a subject of significant interest in the recent literature. One approach has been to study the effect of a single gene on outcomes after treatment for NSCLC. However, several molecular events are known to be associated with the progression of NSCLC, and it is unlikely that a single genetic aberration by itself will account for the clinical behavior of a cancer. Allelic loss, which is one of the common genetic events involved in tumorigenesis and progression, is accumulated throughout the process of tumor evolution. ${ }^{4,5}$

This study was designed to analyze allelic loss (and therefore mutational change) in primary tumors and histologically positive N1 lymph nodes in patients with stage II lung cancer who had undergone pulmonary resection. We selected patients with stage II NSCLC (on the basis of N1 disease) because these patients are usually treated with surgical intervention alone but still have a high incidence of recurrent disease. Because this patient group is characterized by involvement of N1 lymph nodes, we believed that allelic loss within the lymph nodes would play an important role in determining survival. We hypothesized that those patients with more severe mutational changes in lymph nodes would be expected to have poorer outcomes.

\section{Materials and Methods}

The archived paraffin blocks of 40 patients with stage II NSCLC were studied after obtaining institutional review board approval. All patients had stage II disease on the basis of pathologically involved N1 lymph nodes. We excluded patients with T3N0 tumors. Additional inclusion criteria were the presence of adenocarcinoma or squamous carcinoma only. All patients underwent a standard pulmonary resection (lobectomy or pneumonectomy). We excluded patients who had limited resections, such as a wedge or segmental resection.

A minimum of 2 sites within the primary tumor and 1 involved NI lymph node were studied. The most anaplastic areas, as assessed on light microscopy, were selected for microdissection. In the lymph nodes cancer cells are cohesive and tend to form nodular aggregates, and therefore it was relatively easy for the pathologists to microdissect pure metastatic tumor. In the case of primary tumor, cancer cell nests could occasionally be embedded in fibrous tissue or admixed with inflammatory cells, and therefore it was possible that pure tumor was not obtained. However, because the primary tumor was usually large enough, it was possible to avoid these areas, with the purity of all microdissected specimens estimated to be greater than $90 \%$. Additionally, an area of normal parenchyma was also studied to serve as an internal control and to assess for allelic dropout. ${ }^{6}$ Microdissection was performed at all these sites, obtaining 4- $\mu \mathrm{m}$-thickness and 2- to 6-mm- diameter sections of tissue by using methods previously described. ${ }^{7}$

After digestion of the microdissected tissue with proteinase $\mathrm{K}$, aliquots of sampled tissue were used in individual polymerase chain reaction amplification reactions targeting microsatellites situated in proximity to genes of interest. The 16-microsatellite markers chosen were located on 9 chromosomal loci (CLs). These loci are situated within or adjacent to 9 known tumor suppressor genes or oncogenes potentially involved in lung carcinogenesis. The relationship of microsatellite to CLs and genes are indicated in Table 1. The use of more than one microsatellite marker provided a higher yield of information for each CL.

\section{Loss of Heterozygosity Analysis}

Determination of allelic loss was carried out by using phosphorous 33 autoradiography, as previously described. ${ }^{7}$ Allelic dropout was evaluated by imposing a requirement that all normal lung samples must show equal band intensity for the microsatellite being tested on autoradiography. Any patient not showing this was rejected for assessment of allelic loss. Therefore if, in a hypothetic case, the normal lung demonstrated equal band intensity in 8 of the 9 CLs, then 8 CLs were informative.

Loss of heterozygosity ( $\mathrm{LOH}$ ) was determined for both the tumor and lymph node metastases. If there was a diminished band intensity of at least one half the other corresponding bands for CLs that were informative, then $\mathrm{LOH}$ was demonstrated. Analysis of microsatellites provided increased information about each CL. As long as LOH was demonstrated in one of the microsatellites related to a particular CL, then that CL was classified as demonstrating LOH. Additionally, as described above, at least 2 primary tumor sites and 1 lymph node site were microdissected for each patient. In some cases heterogeneity was observed in these different sampled sites. If $\mathrm{LOH}$ was present in at least one of the microdissected sites, then $\mathrm{LOH}$ was recorded as being present for the node or tumor being examined. Two observers evaluated $\mathrm{LOH}$ independently for each specimen, with a concordance ratio of positive $\mathrm{LOH}$ of $100 \%$.

\section{Fractional Allelic Loss and Determination of Risk Group}

Determination of the $\mathrm{LOH}$ and informative rate allowed calculation of the fractional allelic loss (FAL). This was defined as the $\mathrm{LOH}$ of the tumor or node divided by the number of informative CLs in the associated normal lung. By using the same hypothetic case described above as an example, if analysis of a primary tumor demonstrates $\mathrm{LOH}$ in $4 \mathrm{CLs}$, and analysis of the node demonstrates $\mathrm{LOH}$ in $2 \mathrm{CLs}$, then the FAL for the primary tumor is 0.5 (ie, 4/8), and the node is 0.25 (ie, 2/8).

Stratification into risk groups was then made by dividing the FAL in the node by the FAL in the tumor. Because a ratio of 1 or more indicated mutational damage that was as severe or worse in 
TABLE 2. Informative rate and overall frequency of $\mathrm{LOH}$ of each microsatellite

\begin{tabular}{clccc}
\hline $\begin{array}{l}\text { Chromosome } \\
\text { locus }\end{array}$ & Microsatellite & $\begin{array}{c}\text { Informative } \\
\text { rate (n) }\end{array}$ & $\begin{array}{c}\text { LOH in primary } \\
\text { tumor (n) }\end{array}$ & $\begin{array}{c}\text { LOH in lymph } \\
\text { node (n) }\end{array}$ \\
\hline \multirow{2}{*}{$1 \mathrm{p}$} & $D 1 S 407$ & $70 \%(28 / 40)$ & $43 \%(12 / 28)$ & $41 \%(11 / 27)$ \\
& MYCL1 & $78 \%(31 / 40)$ & $58 \%(18 / 31)$ & $45 \%(1 / 31)$ \\
$3 p$ & $D 3 S 1539$ & $78 \%(31 / 40)$ & $61 \%(19 / 31)$ & $52 \%(16 / 31)$ \\
& $D 3 S 2303$ & $80 \%(32 / 40)$ & $66 \%(21 / 32)$ & $52 \%(16 / 31)$ \\
$5 q$ & $D 5 S 592$ & $68 \%(27 / 40)$ & $48 \%(13 / 27)$ & $37 \%(10 / 27)$ \\
& $D 5 S 615$ & $80 \%(32 / 40)$ & $56 \%(18 / 32)$ & $45 \%(14 / 31)$ \\
$7 q$ & $M C C$ & $48 \%(19 / 40)$ & $47 \%(9 / 19)$ & $47 \%(9 / 19)$ \\
$8 q$ & $D 7 S 1530$ & $63 \%(25 / 40)$ & $48 \%(12 / 25)$ & $36 \%(9 / 25)$ \\
$9 p$ & $D S S 373$ & $69 \%(27 / 39)$ & $44 \%(12 / 27)$ & $26 \%(7 / 27)$ \\
& $D 9 S 254$ & $65 \%(26 / 40)$ & $69 \%(18 / 26)$ & $58 \%(15 / 26)$ \\
$10 q$ & $D S S 251$ & $73 \%(29 / 40)$ & $53 \%(14 / 29)$ & $38 \%(11 / 29)$ \\
$17 p$ & $D 10 S 1173$ & $80 \%(32 / 40)$ & $83 \%(19 / 23)$ & $38 \%(12 / 32)$ \\
& $D 10 S 520$ & $58 \%(23 / 40)$ & $30 \%(11 / 37)$ & $65 \%(15 / 23)$ \\
$18 q$ & $D 17 S 1163$ & $95 \%(38 / 40)$ & $74 \%(17 / 23)$ & $32 \%(12 / 37)$ \\
& $T P 53$ & $58 \%(23 / 40)$ & $62 \%(13 / 21)$ & $65 \%(15 / 23)$ \\
\end{tabular}

the nodal metastasis, these patients were classified as high risk. All other patients were classified as low risk. For our hypothetic case, the FAL ratio would be 0.5 (ie, low risk).

\section{Statistical Analysis}

All data were entered into an SPSS (version 10.0 for Windows) file. Statistical analysis included $t$-test analysis of mean values and Kaplan-Meier survival analysis with Breslow tests to compare groups.

\section{Results}

The forty patients included 28 men and 12 women. The median age was 68 years (range, $42-85$ years). The operations were performed over a 4-year period from 1994 to 1998. The operations performed included 26 lobectomies and 14 pneumonectomies. Final histologies were squamous carcinoma in 22 patients and adenocarcinoma in 18 patients, with 8 stage IIa and 32 stage IIb tumors. Median follow-up was 30 months (range, 1-81 months).

The informativeness and frequency of LOH of each microsatellite are shown in Table 2. Informativeness ranged from as low as $48 \%$ to as high as $95 \%$. The mean FALs for the tumor and nodal metastases are demonstrated in Table 3. Mean FAL in both tumor and nodal tissue was significantly higher for squamous cancers than for adenocarcinomas. However, median survival for squamous carcinomas and adenocarcinomas were similar at 35 and 39 months, respectively $(P=.65)$. Median survivals were also similar for stage IIa versus IIb disease (38 vs 39 months, $P=.96$ ) and for patients who underwent lobectomy compared with pneumonectomy (30 months for lobectomy; median survival was not reached for pneumonectomy; $P=.84$ ).

There were 20 high-risk patients and 20 low-risk patients. Median survival was not reached in the low-risk
TABLE 3. Fractional allele loss in adenocarcinoma and squamous carcinoma

\begin{tabular}{lcc}
\hline & $\begin{array}{c}\text { FAL in primary } \\
\text { tumor }\end{array}$ & $\begin{array}{c}\text { FAL in node } \\
\text { metastasis }\end{array}$ \\
\hline $\begin{array}{l}\text { Squamous carcinoma } \\
(\mathrm{n}=22)\end{array}$ & 0.7 & 0.63 \\
$\begin{array}{c}\text { Adenocarcinoma } \\
(\mathrm{n}=18)\end{array}$ & 0.41 & 0.31 \\
$\begin{array}{c}\text { Significance } \\
\text { (squamous } \\
\text { carcinoma vs } \\
\text { adenocarcinoma) }\end{array}$ & $P<.01$ & $P<.01$ \\
\hline
\end{tabular}

$F A L$, Fractional allele loss.

patients and was 35 months for the high-risk patients. This was not significantly different $(P=.3)$. Median disease-free survival (DFS) was not reached in low-risk patients and was 24 months in high-risk patients. This was not significantly different $(P=.36)$

When looking at the adenocarcinomas and squamous carcinomas separately, we found that $9(41 \%)$ of 22 of the squamous carcinomas and $11(61 \%)$ of 18 of the adenocarcinomas were in high-risk patients. Survival and DFS were not significantly different for low-risk and high-risk squamous cell cancers (Figures 1 and 2). Analysis of the patients with adenocarcinoma revealed significant differences between low-risk and high-risk tumors. These are shown in Figures 3 and 4. There were no deaths in the low-risk patients with adenocarcinoma compared with a median survival of 25 months in the high-risk patients with adenocarcinoma $(P=.01)$. The median DFS was not reached for the low-risk patients with adenocarcinoma compared with 14 months for the high-risk patients $(P=.05)$. 


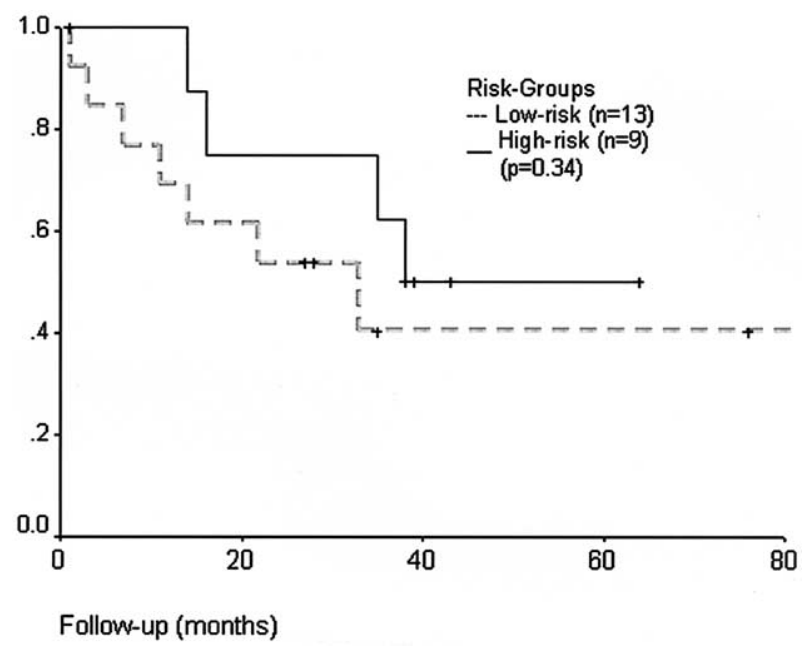

Figure 1. Survival in patients with squamous carcinomas.

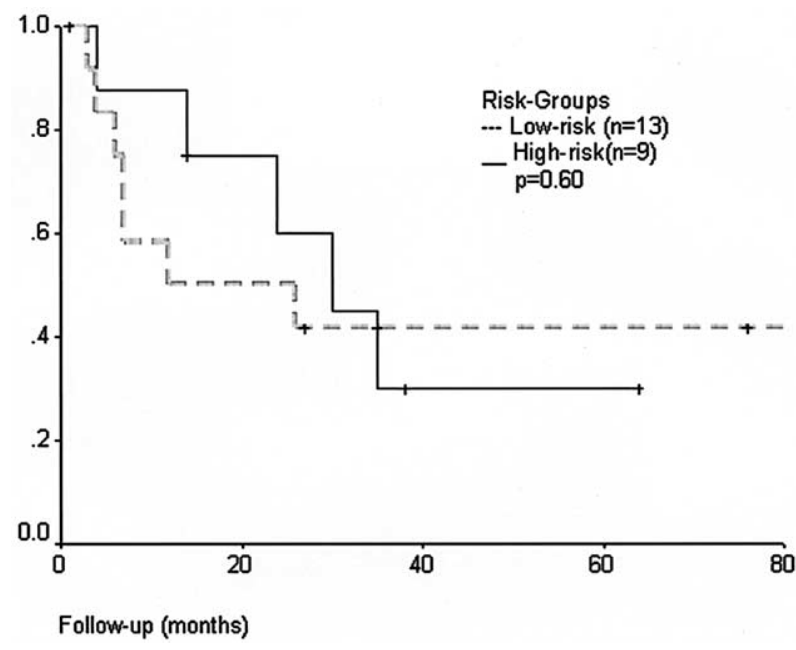

Figure 2. DFS in patients with squamous cell carcinoma.

\section{Discussion}

Stage II NSCLC is usually treated with surgical intervention alone. However, the relatively low survival compared with that seen in patients with stage I NSCLC and the high incidence of systemic metastasis after recurrence ${ }^{8}$ suggest the need for additional therapy. Adjuvant therapies have not met with success. $^{2,3}$ Prognostic markers that could successfully stratify patients might allow us to more effectively direct therapy.

Molecular staging techniques to assess prognosis are usually performed for early stage disease, typically in patients with stage I disease. ${ }^{9}$ Patients with more advanced tumors are considered to have a dismal prognosis, regardless of the status of the biologic marker investigated, ${ }^{10}$ and have not been studied to the same degree. One approach in early stage NSCLC has been to study histologically negative mediastinal lymph nodes for the presence of occult micrometastatic disease. ${ }^{11}$ Another approach that has been

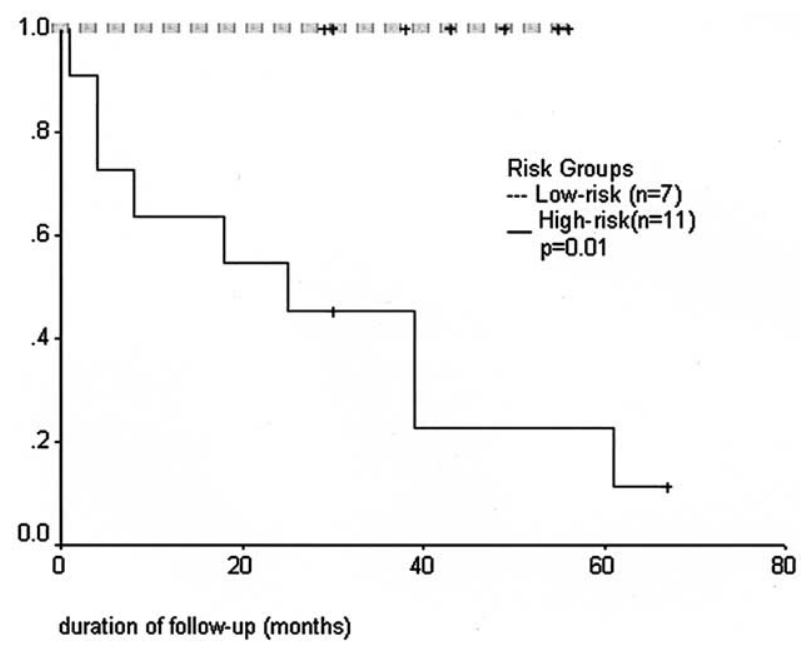

Figure 3. Survival in patients with adenocarcinoma.

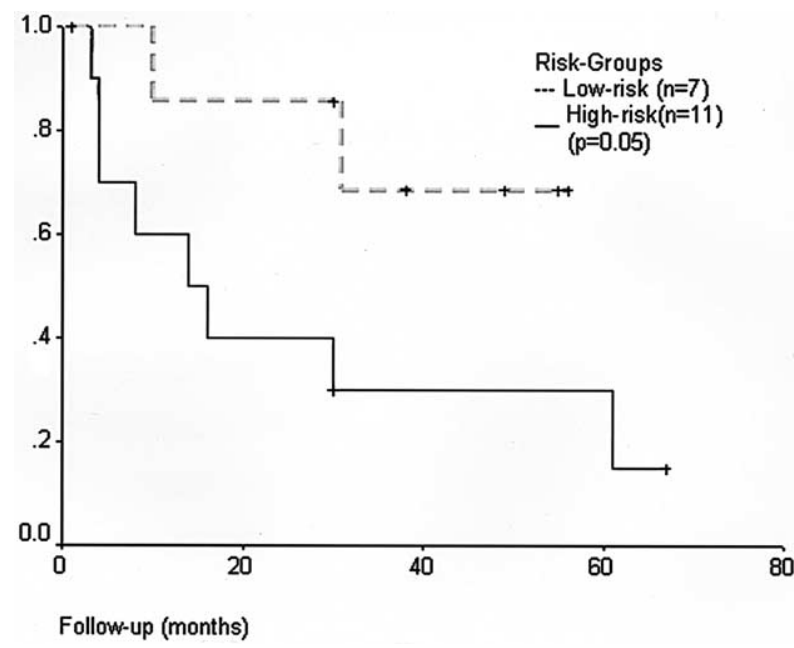

Figure 4. DFS in patients with adenocarcinoma.

described is the use a panel of molecular markers to stratify patients rather than a single-marker approach. ${ }^{10}$ In a study of 408 patients with stage I NSCLC using 10 markers, significant differences in survival were seen for those patients with 6 to 9 markers compared with that seen in those with 0 to 3 markers present. ${ }^{10}$

We elected to use a panel approach in this study. Because all these patients had histologically involved lymph nodes, we hypothesized that the mutational changes in these lymph nodes would allow us to predict biologic behavior. We have previously demonstrated that no single $\mathrm{LOH}$ in the primary tumor is associated with prognosis in stage II NSCLC. ${ }^{12}$ However, $\mathrm{LOH}$ at CLs $3 p$ and $5 q$ in the lymph node is associated with decreased survival. ${ }^{12}$ This supports the view that the FAL of lymph nodes plays an important role when $\mathrm{N} 1$ disease is present.

Previous studies have reported the higher frequency of loss of chromosomal arms in squamous carcinomas compared with 
that in adenocarcinomas. ${ }^{13,14}$ Our data support these findings of differences in the genetic profiles between adenocarcinomas and squamous carcinomas. Squamous carcinomas had a higher FAL in both lymph nodes and in primary tumors. However, survival between squamous carcinomas and adnocarcinomas was similar despite these genetic differences.

Our hypothesis was that if mutational changes were more severe in the lymph nodes compared with in the primary tumor (determined on the basis of the FAL ratio), this would predict behavior. Although there was a trend for worse survival in patients who had an FAL ratio of 1 or more, this was not statistically significant. Further analysis revealed that the differences lay within the adenocarcinoma group. When squamous carcinomas were excluded, the difference between lowrisk and high-risk patients became statistically significant, with no deaths in the low-risk adenocarcinoma group. Thus it appears that the patients with low-risk stage II adenocarcinomas appear to be a subset with favorable outcomes.

The explanation for these differences is unclear. Perhaps the greater LOH seen in squamous carcinomas overall makes comparison between the lymph nodes and primary tumors less meaningful. The behavior of adenocarcinoma appears to be more variable and might depend on the pattern of $\mathrm{LOH}$. Although not studied here, the $\mathrm{LOH}$ profile in primary adenocarcinoma might predict outcomes in stage I tumors. By the time N1 disease has occurred, the lymph node appears to be the critical factor rather than the primary tumor.

In conclusion, we have demonstrated differences in $\mathrm{LOH}$ between squamous carcinomas and adenocarcinomas. Both survival and DFS were improved in low-risk stage II adenocarcinomas on the basis of the FAL ratio between the lymph node and the primary tumor. Larger studies will be needed to confirm the findings of this pilot study.

\section{References}

1. Mountain CF. Revisions in the international system for staging lung cancer. Chest. 1997;112(suppl):239S-41S.

2. Holmes EC, Gail M for the Lung Cancer Study Group. Surgical adjuvant therapy for stage II and stage III epidermoid cancer of the lung. N Engl J Med. 1986;315:1377-81.

3. Holmes EC. Surgical adjuvant chemotherapy for stage II and stage III adenocarcinoma and large cell undifferentiated carcinoma. Chest. 1994;106(suppl):293S-6S.

4. Vogelstein B, Fearon ER, Hamilton SR, Kern SE, Preisinger AC, Leppert M, et al. Genetic alterations during colorectal-tumor development. $N$ Engl J Med. 1988;319:525-32.

5. Sato T, Tanigami A, Yamaakawa K, Akiyama F, Kasumi F, Sakamoto G, et al. Allelotype of breast cancer. Cumulative allele loss promote tumor progression in primary breast cancer. Cancer Res. 1990;50:7184-9.

6. Dreesen JC, Bras M, Coonen E, Dumolin JC, Evers JL, Geraedts JP. Allelic dropout caused by allele-specific amplification failure in singlecell PCR of the cystic fibrosis delta F508 deletion. J Assist Reprod Genet. 1996;13:112-4.

7. Rao UNM, Baker A, Swalsky PA, Finkelstein SD. Max interacting protein 1: Loss of heterozygosity is frequent in desmoplastic melanoma. Mod Pathol. 1999;12:344-50.

8. Socinski MA. The current status of adjuvant chemotherapy for resected non-small cell lung cancer. Semin Oncol. 1999;26(suppl 15):27-33.

9. Kwaitkowski DJ, Harpole DH, Godleski J, Herndon JE, Shieh D, Richards W, et al. Molecular pathological substaging in 244 stage I non-small cell cancer patients: Clinical implications. J Clin Oncol. 1998;16:2468-77.

10. D'Amico T. Molecular biological substaging of non-small cell lung cancer. J Thorac Cardiovasc Surg. 2002;123:409.

11. D'Cunha J, Corfits AL, Herndon JE, Kern JA, Kohman LJ, Patterson AJ, et al. Molecular staging of lung cancer: Real-time polymerase chain reaction estimation of lymph node micrometastatic tumor cell burden in stage I non-small cell lung cancer-preliminary results of Cancer and Leukemia Group B Trial 9761. J Thorac Cardiovasc Surg. 2002;123:484-91.

12. Sasatomi E, Finkelstein SD, Woods JD, Bakker A, Swalsky PA, Luketich JD, et al. Comparison of accumulated allele loss between primary tumor and lymph node metastasis in stage II non-small cell lung carcinoma: implications for the timing of lymph node metastasis and prognostic value. Cancer Res. 2002;62:2681-9.

13. Sato S, Nakamura Y, Tscuchiya E. Difference of allelotype between squamous cell carcinoma and adenocarcinoma of the lung. Cancer Res. 1994;54:5652-5.

14. Tscuchiya E, Nakamura Y, Weng SY, Nakagawa K, Tschiya S, Sugano $\mathrm{H}$, et al. Allelotype of non-small cell lung carcinoma-comparison between loss of heterozygosity in squamous cell carcinoma and adenocarcinoma. Cancer Res. 1992;52:2478-81.

\section{Discussion}

Dr Steven J. Mentzer (Boston, Mass). If the cells that are in the lymph node have an $\mathrm{LOH}$ and the mutation reflects the $\mathrm{LOH}$ observed in different parts of the tumor, then isn't it simply a selection issue? In other words, couldn't active selection within the node produce this subset of the tumor found in the lymph node?

Dr Fernando. It might be true that we are seeing a subset of tumor within the lymph node and that this might be a sampling issue. However, in some cases we are seeing a different and more severe pattern of $\mathrm{LOH}$ in the involved lymph node compared with in the primary tumor, which is why we believe the node is important for predicting clinical behavior.

Dr David R. Jones (Charlottesville, $\mathrm{Va}$ ). Did you look at the histologic grade of the tumor? Is your analysis of mutational alterations in the tumor any better than evaluating whether the tumor is well differentiated, poorly differentiated, or moderately differentiated?

Finally, which loci do you currently recommend analyzing? All 9? Or were there 3 or 4 that you were able to identify as the main players when you analyzed your LOH analysis?

Dr Fernando. There were 2 CLs in the lymph nodes that were associated with poor prognosis. These were $3 p$ and $5 q$. When we looked at the primary tumor, we could not identify any CL that was associated with prognosis.

Dr Steven J. Mentzer, (Boston, Mass). So you did not find any evidence of active selection within the lymph node as opposed to just passive embolic spread of the tumor? Was there anything that would suggest to you that you had active selection; that is, that the immune system was recognizing the tumor within the lymph node?

Dr Fernando. No, active selection within the lymph node was not addressed in this study.

Dr S. Mentzer. Thank you very much. 